Article

\title{
A Novel Bearing Lubricating Device Based on the Piezoelectric Micro-Jet
}

\author{
Kai Li ${ }^{1}$, Junkao Liu ${ }^{1}$, Weishan Chen ${ }^{1, *}$, Lei Ye ${ }^{1}$ and Lu Zhang ${ }^{2}$ \\ 1 State Key Laboratory of Robotics and System, Harbin Institute of Technology, Harbin 150001, China; \\ sdcxlikai@126.com (K.L.); jkliu@hit.edu.cn (J.L.); yelei6833@126.com (L.Y.) \\ 2 AVIC, Harbin Dongan Engine Corporation LTD, Harbin 150001, China; zhanglu916hit@163.com \\ * Correspondence: cws@hit.edu.cn; Tel./Fax: +86-451-8641-6119
}

Academic Editor: Sheng-Yuan Chu

Received: 15 December 2015; Accepted: 21 January 2016; Published: 1 February 2016

\begin{abstract}
A novel bearing lubricating device, which is embedded in gyroscope's bearing system and based on the theory of the piezoelectric micro-jet, was designed for this study. The embedded structure of a bearing lubricating system can make effective use of the limited space of bearing systems without increasing the whole mass of the system. The drop-on-demand (DOD) lubrication can be realized by the piezoelectric micro-jet system to implement the long acting lubrication of the bearing system. A mathematical model of inlet boundary conditions was established to carry on the numerical simulation based on CFD. The motion states of the droplets with different voltage excitations were analyzed via numerical simulations, and the injection performances of the piezoelectric micro-jet lubricating device were tested in accordance with past experiments. The influences of different parameters of voltage excitation on injection performance were obtained, and the methods of adjusting the injection performance to meet different requirements are given according to the analyses of the results. The mathematical model and numerical simulation method were confirmed by comparing the results of past simulations and experiments.
\end{abstract}

Keywords: piezoelectric micro-jet; bearing lubrication; embedded structure; liquid injection

\section{Introduction}

The drop-on-demand (DOD) injection technology, which was first proposed in ink-jet printing technology, has the advantages of higher injection precision, better control flexibility and greater efficiency than continuous injection [1]. As the primary structure forms to realize DOD injection, the piezoelectric micro-jet brings about high speed development and widespread applications in 2-D or 3-D printing [2], bio-medical field [3-5], material science [6], MEMS [7], electric fuel injection technology $[8,9]$ etc.

Due to the development of the electronic technology, the main factor that restricts the life of space system has gradually turned into the bearing lubrication failure [10]. Researchers are mainly engaged in the research of new additives for lubricating oil in order to improve its effective lifetime [11-13] or design new friction structures [14]. To some extent, these solutions increase the longevity of the friction structure, but we do not think it can fundamentally solve the problem of deterioration, leakage and pollution of lubricating oil.

To achieve controllable, quantitative and long-acting lubrication of the gyroscope's bearing system, a novel bearing lubricating device, based on the theory of the piezoelectric micro-jet, was proposed for this study. Piezoelectric micro-jets are embedded in the bearing system so as not to increase the overall quality and space. For this study, we only designed one kind of structure of the micro-jet to do the research, and the influences of different structure forms and the number of micro-jets in the results will be carried 
out in future works. The injection performances of the micro-jet with different parameters of voltage excitation were studied here, and the relation curves of the performance parameter with related influencing parameters were determined by the results of our numerical simulations and experiments. The results indicate that the piezoelectric micro-jet lubricating device possesses excellent feasibility and effectiveness, which proves that the device can be used as an effective method for the lubrication of bearing systems.

\section{Structure and Operating Principle}

The structure of the piezoelectric micro-jet lubricating device, which was designed for this study, mainly consists of a piezoelectric vibrator and a nozzle, as shown in Figure 1. The vibrator vibrates when pulse voltages are applied to it, while, at the same time, acoustic pressure waves are created and spread through the liquid in the cavity of the micro-jet, and droplets are jetted out of the cavity when the pressure waves spread to the nozzle. This is how the piezoelectric micro-jet works.

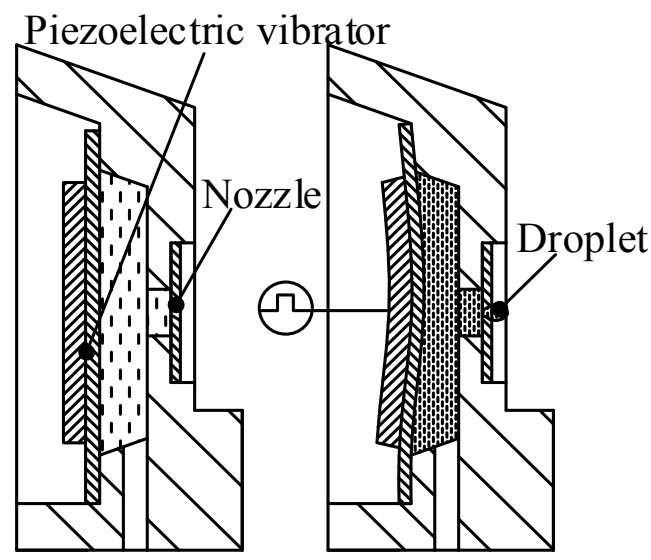

Figure 1. The structure of the micro-jet.

To realize the DOD lubrication of the gyroscope's bearing system, we made full use of the space of the bearing system and embedded the piezoelectric micro-jets into it, as shown in Figure 2. The embedding of the micro-jets cause no change to the overall volume. In order to provide lubricating oil to the cavity of the micro-jet and decrease the total mass of the system, the central spindle was desgined as a hollow craft to be the lubricating oil supply channel such that the lubricating oil is transported to the interior cavity of the micro-jets through the lubricating oil supply channel, and, when the bearing lubrication is insufficient, the droplets of the lubricating oil are sprayed out to the bearings by the pressure waves that are generated by the vibrators.

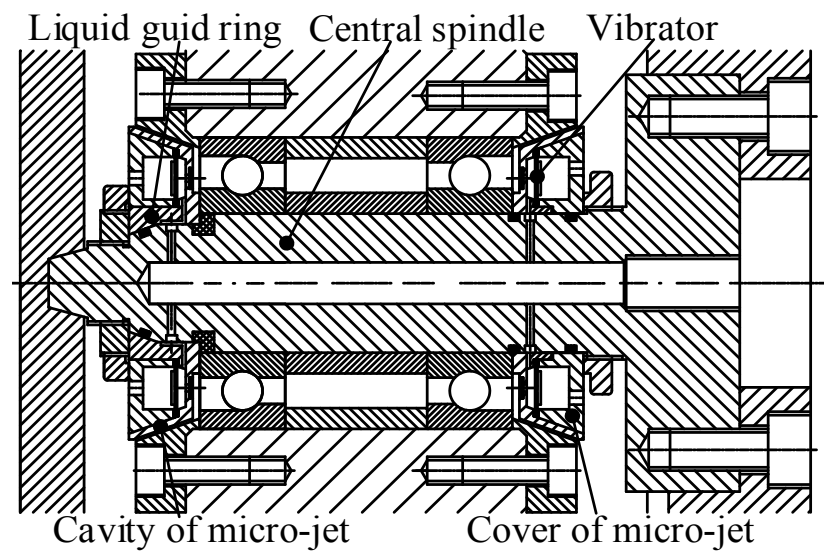

Figure 2. The local structure of gyroscope after embedding the piezoelectric micro-jets. 


\section{Simulation Settings}

Because of the quick response time of the injection process of the micro-jets and the short forming time of the micro-droplets, it was difficult to analyze the characteristics of the droplets, such as the formation time, velocity, molding length and motion state, during the injection process under the existing experimental conditions. Therefore, it was necessary to carry out numerical simulations of the injection process of the droplets in order to analyze the performance of the injection.

\subsection{The Finite Element Model}

The piezoelectric vibrator was composed of piezoelectric ceramic and copper films; they were boned together and made as the two electrodes of the vibrator, respectively. The inner ring diameter of copper film and PZT were $\phi 22 \mathrm{~mm}$ and $\phi 25 \mathrm{~mm}$, respectively. The whole finite element model of the micro-jet is shown in Figure 3a, and, since the micro-jet is a rotating body, we have simplified it here into a two-dimensional model. The main dimension of each part of the micro-jet is shown in Figure 3b,c.
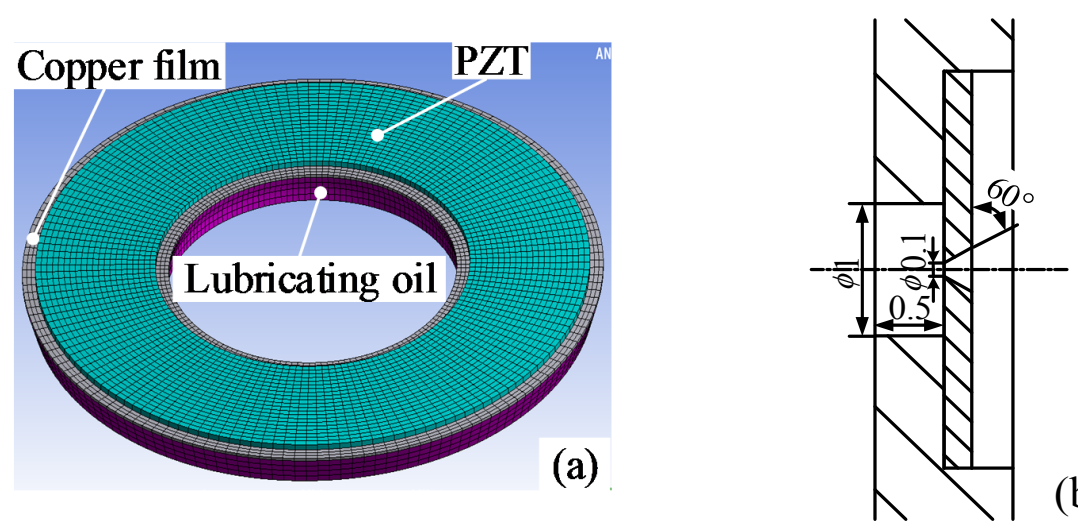

(b)

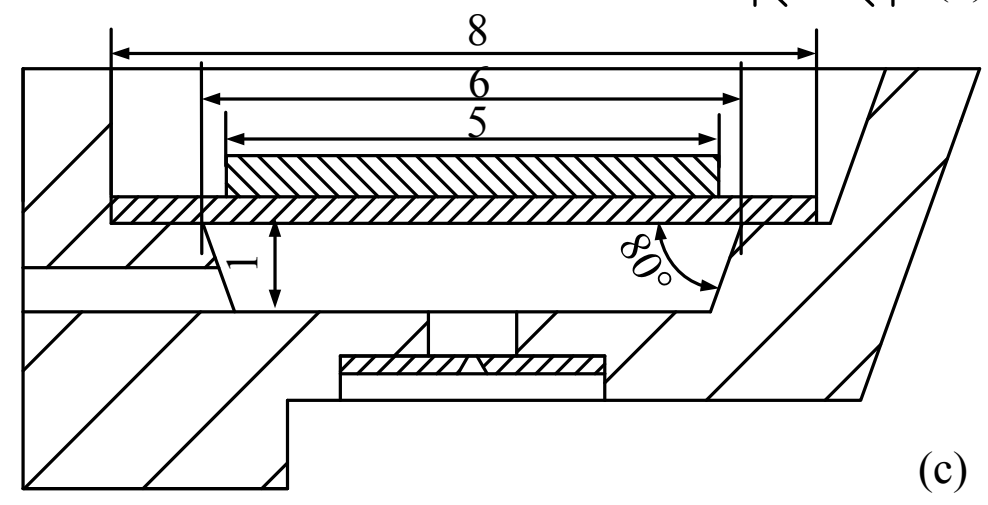

Figure 3. The model and main dimension of the micro-jet (unit: $\mathrm{mm}$ ): (a) the whole finite element model; (b) the nozzle part; (c) the simplified two-dimensional model.

\subsection{The Simulation Setting}

The most suitable working frequency and the displacement-time relations of particles were analyzed by the FEM software ANSYS. The density, elasticity modulus and Poisson ratio of the copper film were set at $7.5 \times 10^{3} \mathrm{~kg} / \mathrm{m}^{3}, 7.65 \times 10^{10} \mathrm{~N} / \mathrm{m}^{2}$ and 0.32 , respectively. The density and dynamic viscosity of the lubrication oil were set at $859 \mathrm{~kg} / \mathrm{m}^{3}$ and $8.91 \times 10^{-3} \mathrm{~Pa} \cdot \mathrm{s}$. The material of the ceramic was PZT-5H, and the physical properties (elastic stiffness constant matrix $\left[{ }^{\mathrm{E}}\right]$, piezoelectric stress constant matrix $[e]$ and dielectric constant matrix $\left.\left[\varepsilon^{\mathrm{T}}\right]\right)$ of the PZT in the simulation were set as: 


$$
\begin{gathered}
{\left[c^{\mathrm{E}}\right]=\left[\begin{array}{cccccc}
13.2 & 7.3 & 7.1 & 0 & 0 & 0 \\
7.3 & 13.2 & 7.1 & 0 & 0 & 0 \\
7.1 & 7.1 & 11.5 & 0 & 0 & 0 \\
0 & 0 & 0 & 2.6 & 0 & 0 \\
0 & 0 & 0 & 0 & 2.6 & 0 \\
0 & 0 & 0 & 0 & 0 & 3
\end{array}\right] \times 10^{10}\left(\mathrm{~N} / \mathrm{m}^{2}\right)} \\
{[e]=\left[\begin{array}{cccc}
0 & 0 & -2.4 \\
0 & 0 & -2.4 \\
0 & 0 & 17.3 \\
0 & 0 & 0 \\
0 & 12.95 & 0 \\
12.95 & 0 & 0
\end{array}\right]\left(\mathrm{C} / \mathrm{m}^{2}\right)} \\
{\left[\varepsilon^{\mathrm{T}}\right]=\left[\begin{array}{cccc}
804.6 & 0 & 0 \\
0 & 804.6 & 0 \\
0 & 0 & 659.7
\end{array}\right] \times 10^{-11}(\mathrm{~F} / \mathrm{m})}
\end{gathered}
$$

$\left[\varepsilon^{\mathrm{T}}\right]=$ Due to the existence of the FSI (fluid-structure interaction) effect in the simulation, the acoustic element Fluid30 was selected as the element type of lubrication oil part, while only the pressure DOF (degree of freedom) was maintained. The element type of the fluid-structure interface was set for Fluid 30 with DOF of $U_{x}, U_{\mathrm{y}}, U_{z}$, and the pressure maintained. The pressure boundary conditions of inlet and outlet of the micro-jet were all set at 0 , and other constraints were set as its actual situation.

\section{The Velocity-Inlet Boundary Conditions}

In order to obtain the velocity-inlet boundary conditions for the injection simulations, we decided to get the volume change function of the cavity of the micro-jet first, which was caused by the vibration of the piezoelectric vibrator.

As we mainly focused on the injection performances of the micro-jet for simplicity's sake, the backward flow occurred at the inlet of ball-race contact, and the thermal balance in the bearing etc., which had effects on the lubrication performances of the bearing, were not considered for this study. For details, please read the article by Mohammadpour et al. [15].

The first four mode shapes of the micro-jet are shown in Figure 4; the corresponding resonant frequencies are $1.83 \mathrm{kHz}, 4.32 \mathrm{kHz}, 7.01 \mathrm{kHz}$ and $9.49 \mathrm{kHz}$, respectively. The frequency of the first mode shape was preferable as the working frequency due to the central symmetrical characteristic, which can reduce the difficulty of the positioning when being assembled.
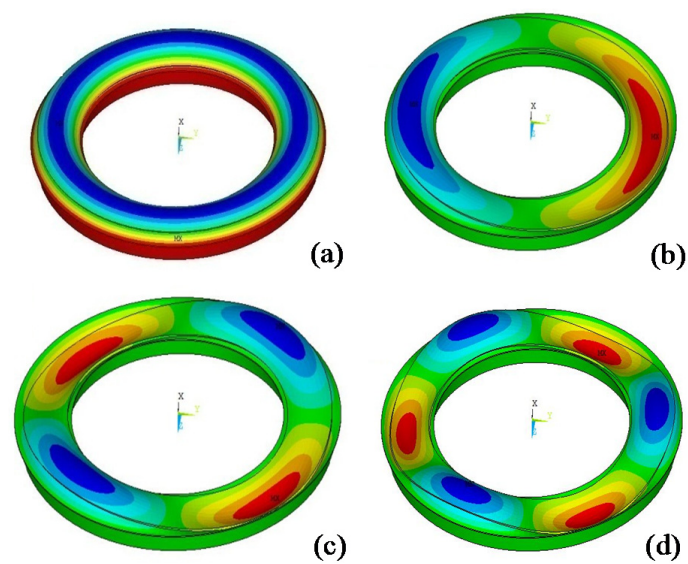

Figure 4. The first four modes of vibration. 
The displacement-time relation curves of particles with different radial positions on the fluid-structure interface were obtained, as shown in Figure 5. The square pulse voltages were selected, as the excitations with the frequency and amplitude were set to $1.83 \mathrm{kHz}$ and $100 \mathrm{~V}$, respectively.

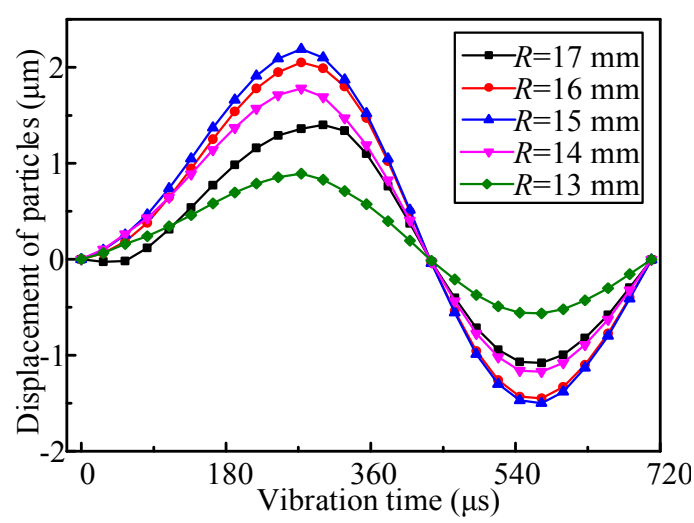

Figure 5. The displacement-time relation curves of particles with different radial position on the fluid-structure interface ( $R$ is the radius of the particle's position).

A numerical fitting model of a Fourier series function was adopted to fit the displacement-time relation curve of the particles with radius $R=15 \mathrm{~mm}$, in accordance with the data of Figure 5, as shown in Equation (1). In the equation, $w / 2 \pi$ is the vibration frequency and $t$ represents the time. The fitting coefficients in the equation above are listed in Table 1.

$$
u_{z}(t)=a_{0}+\sum_{n=1}^{13} a_{n} \cos n w t+\sum_{n=1}^{13} b_{n} \sin n w t, \quad 0 \leqslant t \leqslant 684 \mu \mathrm{s}
$$

Table 1. The coefficients of Equation (1).

\begin{tabular}{cccccc}
\hline $\boldsymbol{n}$ & $\boldsymbol{a}_{n}$ & $\boldsymbol{b}_{n}$ & $\boldsymbol{n}$ & $\boldsymbol{a}_{n}$ & $\boldsymbol{b}_{n}$ \\
\hline 0 & 0.3456 & - & 7 & -0.0204 & -0.0282 \\
1 & -0.4588 & 1.5104 & 8 & -0.0180 & -0.0242 \\
2 & 0.2656 & -0.4576 & 9 & -0.0203 & -0.0145 \\
3 & 0.0208 & -0.0999 & 10 & -0.0211 & -0.0140 \\
4 & -0.0034 & -0.0718 & 11 & -0.0183 & -0.0099 \\
5 & -0.0069 & -0.0491 & 12 & -0.0217 & -0.0057 \\
6 & -0.0122 & -0.0310 & 13 & -0.0211 & -0.0047 \\
\hline
\end{tabular}

The fitting curve, which was determined by Equation (1), gives a better fit for the simulation data, as shown in Figure 6.

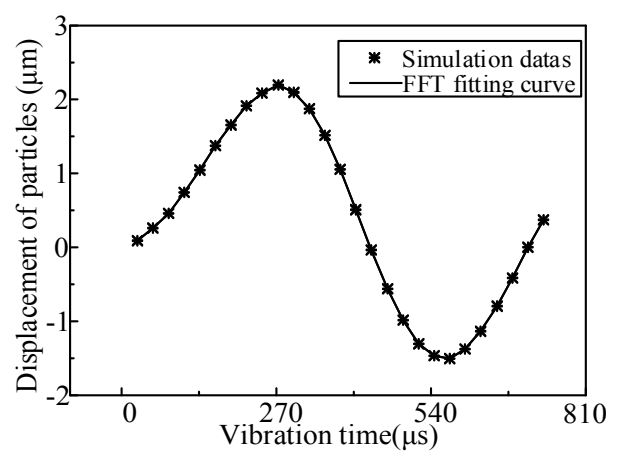

Figure 6. The fitting curve, which was determined by Equation (1). 
The relation curve of vibration amplitude and radial position of particles was obtained from Figure 5, as shown in Figure 7. As can be seen, the curve is approximately axially symmetric, and the symmetry axis is $R=15 \mathrm{~mm}$. Moreover, the vibration directions and frequencies of the particles are all the same. For convenience, we call the particles with radius $R=15 \mathrm{~mm}$ "central line particles."

Combined with Equation (1), the vibration displacement $U_{Z}(t, r)$ of particles on the fluid-structure interface was deduced by fitting the curve in Figure 7, as shown in Equation (2). In this equation, $r$ is the radial distance between particles and the central line particles, $u_{z m 0}$ is the vibration amplitude of the central line particles, and $2 r_{0}$ is the effective radial width of the vibrator (here $r_{0}=3 \mathrm{~mm}$ ). The fitting coefficients in Equation (2) are listed in Table 2.

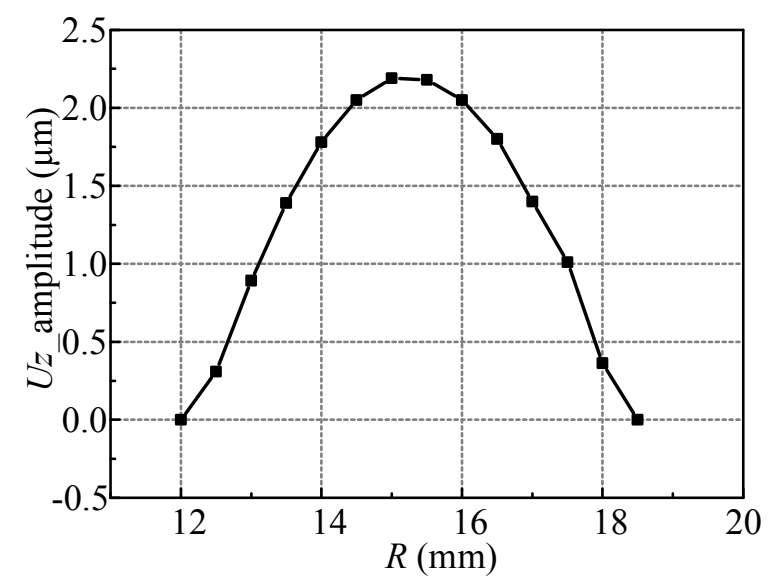

Figure 7. The relation curve of vibration amplitude and radial position of particles.

$$
U_{Z}(t, r)=\left(c_{0}+\sum_{n=1}^{6} c_{n} \cos n w r+\sum_{n=1}^{6} d_{n} \sin n w r\right) \cdot u_{z}(t) / u_{z m 0}, \quad-r_{0} \leqslant r \leqslant r_{0}
$$

Table 2. The coefficients of Equation (2).

\begin{tabular}{cccccc}
\hline $\boldsymbol{n}$ & $\boldsymbol{c}_{n}$ & $\boldsymbol{d}_{n}$ & $\boldsymbol{n}$ & $\boldsymbol{c}_{n}$ & $\boldsymbol{d}_{n}$ \\
\hline 0 & 1.2439 & - & 4 & -0.0076 & -0.0052 \\
1 & -1.0628 & 0.2669 & 5 & -0.0042 & -0.0233 \\
2 & -0.1473 & 0.0921 & 6 & 0.0060 & -0.0200 \\
3 & -0.0326 & 0.0263 & & & \\
\hline
\end{tabular}

The volume change of the cavity $\Delta V(t)$ was deduced, as shown in Equation (3), where $R_{0}$ is the radius of the central line particles.

$$
\Delta V(t)=\int_{-r_{0}}^{r_{0}} 2 \pi\left(R_{0}-r\right) U_{Z}(t, r) d r
$$

Then, the mean volume flow of the inlet of single nozzle was calculated with Equation (4):

$$
q(t)=\frac{1}{2} \cdot \frac{d V(t)}{d t}=\frac{d}{d t} \int_{-r_{0}}^{r_{0}} \pi\left(R_{0}-r\right) U_{Z}(t, r) d r
$$

Therefore, the inlet velocity boundary conditions of the single nozzle is:

$$
v(t)=\frac{4}{A_{1}^{2} \cdot u_{z m 0}} \frac{d}{d t}\left[\int_{-r_{0}}^{r_{0}}\left(R_{0}-r\right)\left(c_{0}+\sum_{n=1}^{7} c_{n} \cos n w r+\sum_{n=1}^{7} d_{n} \sin n w r\right) \cdot u_{z}(t) d r\right]
$$




\section{The Injection Performance of the Nozzle}

In order to get the injection performance of the nozzle under single-pulse voltage, we utlized CFD simulation software (CFD-ACE+, ESI Group) to analyze the forming and moving stages of the droplets. According to the models and dimensions shown in Figure 3, the injection model of the nozzle was simplified in the simulation, as shown in Figure 8.

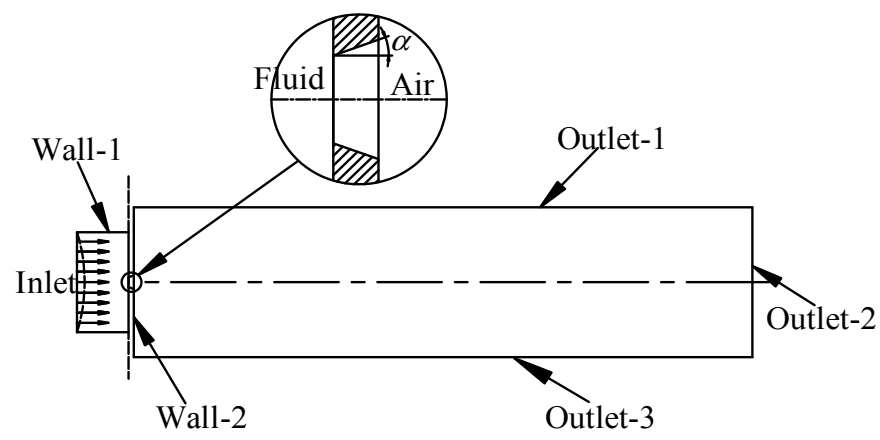

Figure 8. The injection model of the nozzle.

The left and right sides of the nozzle were set as fluid- and air-field respectively, and the divergence angle $\alpha$ is set at $30^{\circ}$ to correspond to the dimensions in Figure 3b. The inner wall of the cavity, Wall-1, is half-hydrophilic, and the contact angle was set at $85^{\circ}$. The air-liquid interface, Wall-2, is hydrophobic, and the contact angle was set at $165^{\circ}$. The velocity-inlet boundary conditions were set in accordance with Equation (5), and others were all set to have no slip boundaries. The density and dynamic velocity of the air were set at $1.1614 \mathrm{~kg} / \mathrm{m}^{3}$ and $1.846 \times 10^{-3}$ respectively, and the surface tension of the lubricating oil was set at $0.0725 \mathrm{~N} / \mathrm{m}$. The temperature was set at $20^{\circ}$, ignoring the influence of gravity. The structured quadrilateral cell was selected in this gas-liquid two-phase flow model, and the analysis type was set to have transient analysis.

In order to improve the uniformity of the lubrication of the bearing, we decided to make the droplets dispersed into a plurality of small droplets, and the smaller the droplets were, the better. The total time used for an ejected droplet from the initial formation to travel completely outside of the nozzle affected the response time of the lubrication, and we called this the "molding time" of the droplets. We also decided that the droplets' molding time should be decreased and that velocity should be increased so as to reduce the response time of the bearing lubricating time. Furthermore, we called the length of the droplet at the end of the molding time the "molding length" of the droplet, determining the minimum effective working distance between the nozzle and the bearing.

Thus, the influences of the voltage pluses on the molding time, velocity, molding length, and dispersion condition of the droplets were analyzed. The major parameters of the pulse voltages were amplitude, duty ratio and frequency, and, since the working frequency was limited by the structure and lubricating oil properties, only the first two parameters' influences were analyzed.

\subsection{The Influence of the Amplitude}

Setting the duty ratio at $50 \%$, and keeping the properties of the lubricating oil unchanged, pulse voltages with different amplitudes were applied to the piezoelectric vibrator. The velocity-inlet boundary conditions were obtained by Equation (5) and then applied to the injection modal.

The changing curves of molding time and velocity corresponding to different amplitudes of the pulse voltage are shown in Figure 9a. As can be seen, with the increase of the voltage amplitude, the molding time decreased and the velocity increased gradually. Thus, we decided that, for a quick response to the lubrication, the amplitude of the pulse voltages should be raised to a certain range.

The relationship curve of molding length and amplitude was obtained, as shown in Figure 9b. It appears that the molding length rose with the increase of the amplitude of the pulse voltages. 
Therefore, when increasing the amplitude of the pulse voltages, we decided to assure that the molding length was shorter than the distance between the nozzle and the bearing.
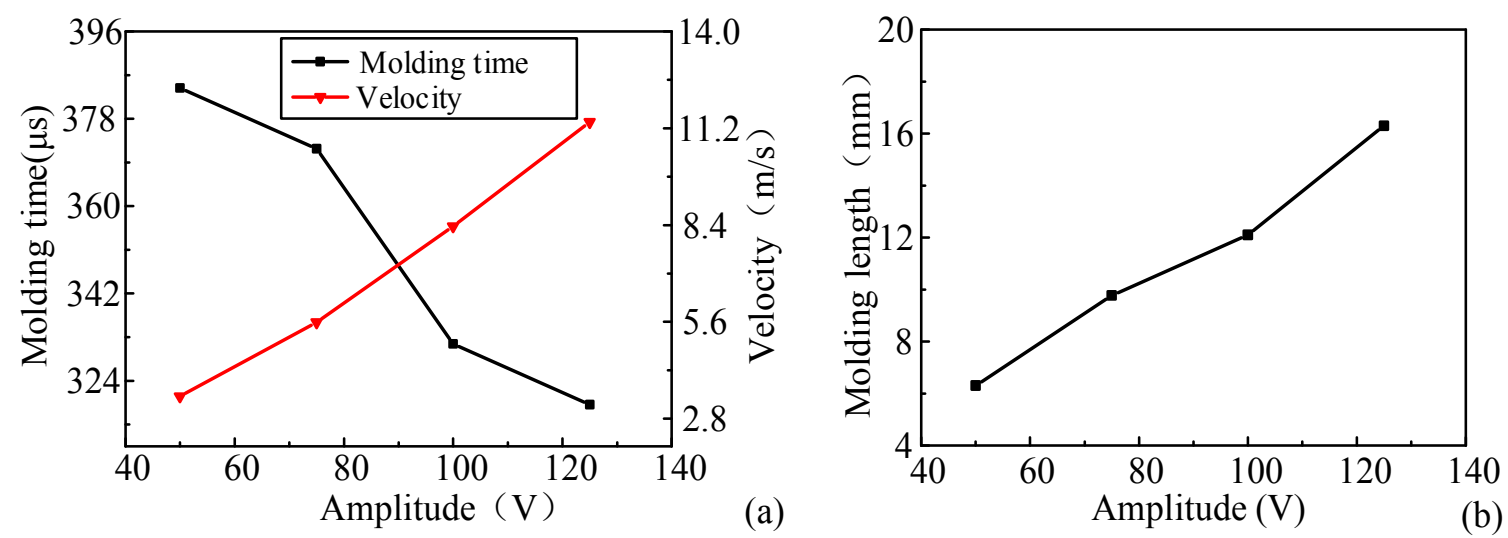

Figure 9. (a) The changing curves of molding time and velocity; (b) the relationship curve of molding length and amplitude.

The dispersion conditions of the droplets with different amplitudes of pulse voltages are shown in Figure 10. It can be seen that, when the voltage amplitude was relatively low, the shape of the droplet was regular and roughly spherical, and, with the increase of the voltage amplitude, the droplet was gradually dispersed; the larger the voltage, the higher the degree of divergence. Thus, in order to achieve sufficient lubrication, we decided that a larger amplitude of the pulse voltages should be selected in a certain range.

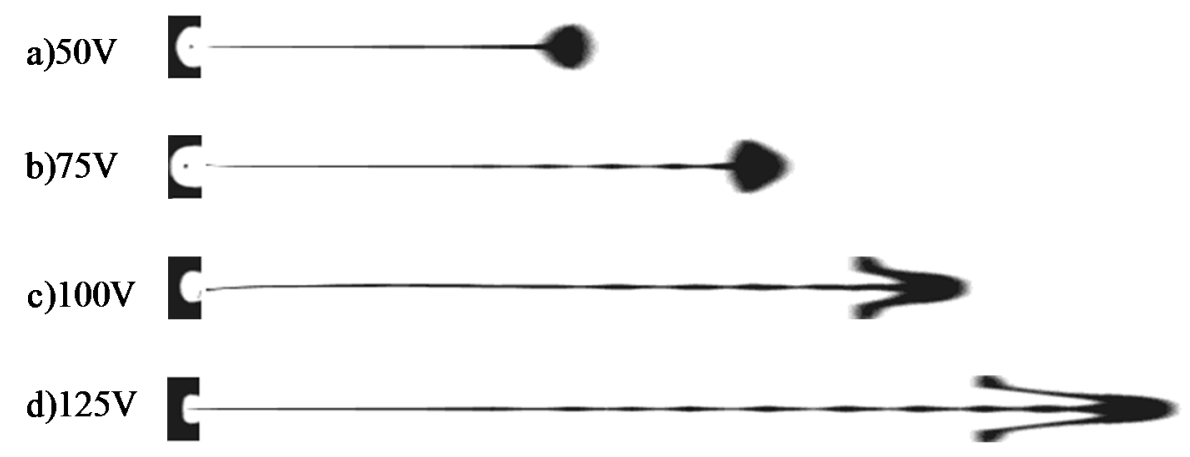

Figure 10. The dispersion conditions of the droplets with different amplitudes.

\subsection{The Influence of the Duty Ratio}

Keeping the amplitude of the pulse voltages at $100 \mathrm{~V}$, the impact of duty ratio on the injection performance was analyzed by changing the ratio size. As before, Equation (5) was used to calculate the velocity-inlet boundary conditions that were applied to the injection modal. To be clear, the duty ratio was set to 1 in the results instead of 0.999 for convenience.

The changing curves of molding time and velocity under pulse voltages with different duty ratios are shown in Figure 11a. The figure demonstrates that the velocity reduced along with the increase of the duty ratio, while the molding time gradually increased. Therefore, we concluded that, in a certain range, the duty ratio of the pulse voltages should be raised so as to improve the response time of the lubrication.

The relationship curve of the molding length and duty ratio was obtained, as shown in Figure 11b. As we can see, with the increase of the duty ratio of the pulse voltages, the molding length rose gradually. Figure $11 \mathrm{~b}$ has a guiding role for determining the minimum distance between the bearing and the nozzle when different duty ratios are selected. 

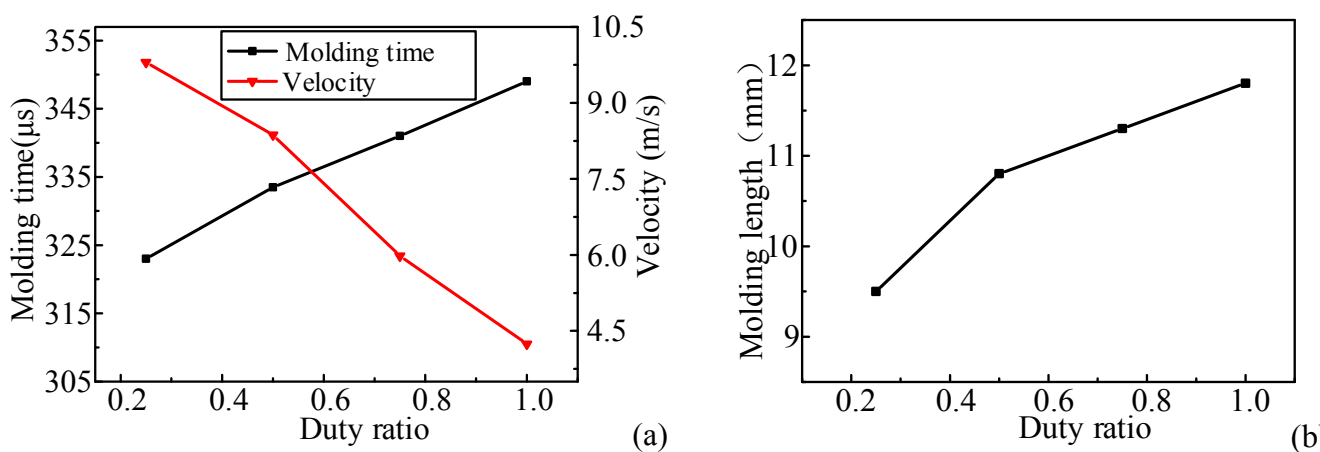

Figure 11. (a) The changing curves of molding time and velocity; (b) the relationship curve of molding length and duty ratio.

The dispersion conditions of the droplets with different duty ratios of pulse voltages are shown in Figure 12. The figure shows that the droplet is roughly one spherical drop when relatively low duty ratio is applied, and the level of the dispersion increases with the increase of the duty ratio. Therefore, we concluded that, for achieving sufficient lubrication, a larger duty ratio should be selected if conditions are met.

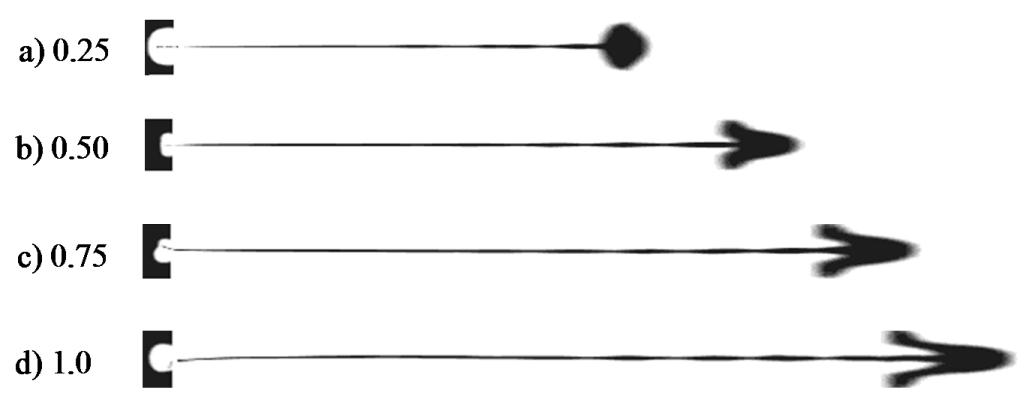

Figure 12. The dispersion conditions of the droplets with different duty ratio.

\section{The Experiment Performance of the Micro-Jet}

In order to analyze the experiment performance of the piezoelectric micro-jet lubricating system, a simple experimental device was manufactured and assembled on the basis of design drawings, as shown in Figure 13. The bearings to be lubricated were removed so as to improve the convenience of testing, and the parameters of the lubricating oil used in the experiment were the same as in the simulation. We were careful to empty the air bubbles in the cavity of the micro-jet, as the existence of bubbles would have seriously affected the accuracy of the results.
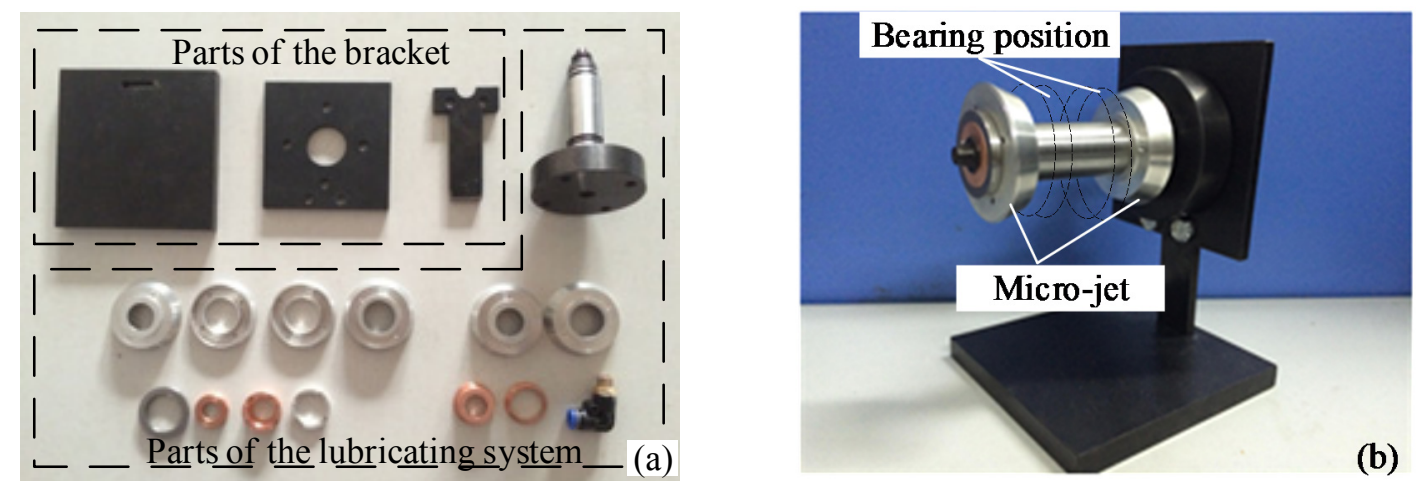

Figure 13. The experimental device of the piezoelectric micro-jet lubricating system: (a) The parts of the device; (b) The assembly of the device. 


\subsection{Experiment Study of the Working Frequency}

The experimental platform of the working frequency test mainly consisted of a PC, a scanning Vibrometer (PSV-400-M2) and a power amplifier, as shown in Figure 14. The actual vibration mode and corresponding frequency of the device were obtained through this experimental platform, and the most appropriate practical working frequency was selected in accordance with the analysis of the experimental results.

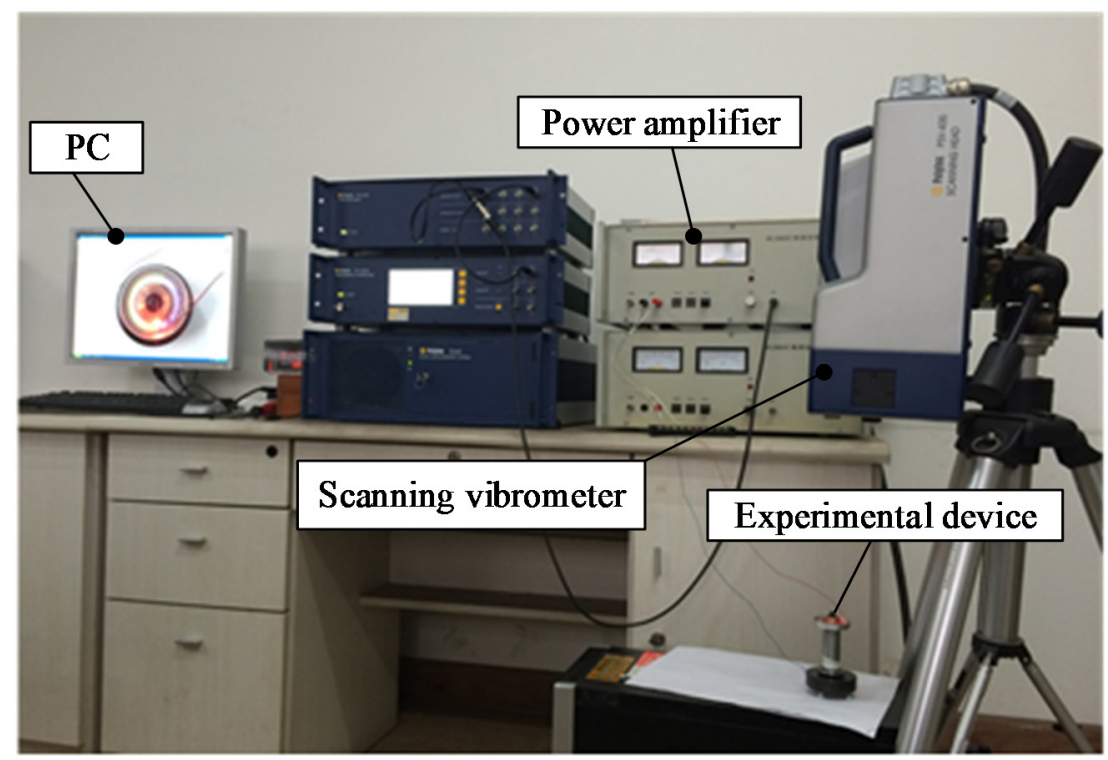

Figure 14. The experimental platform of working frequency test.

In this experiment, the amplitude of the pulse voltages was set at $100 \mathrm{~V}$, and the properties of the lubricating oil filled in the cavity of the micro-jet were the same as what was set in the simulation. The vibration velocity response spectrum and first-order vibration shape of the piezoelectric vibrator were obtained by the scanning vibrometer, as shown in Figure 15.
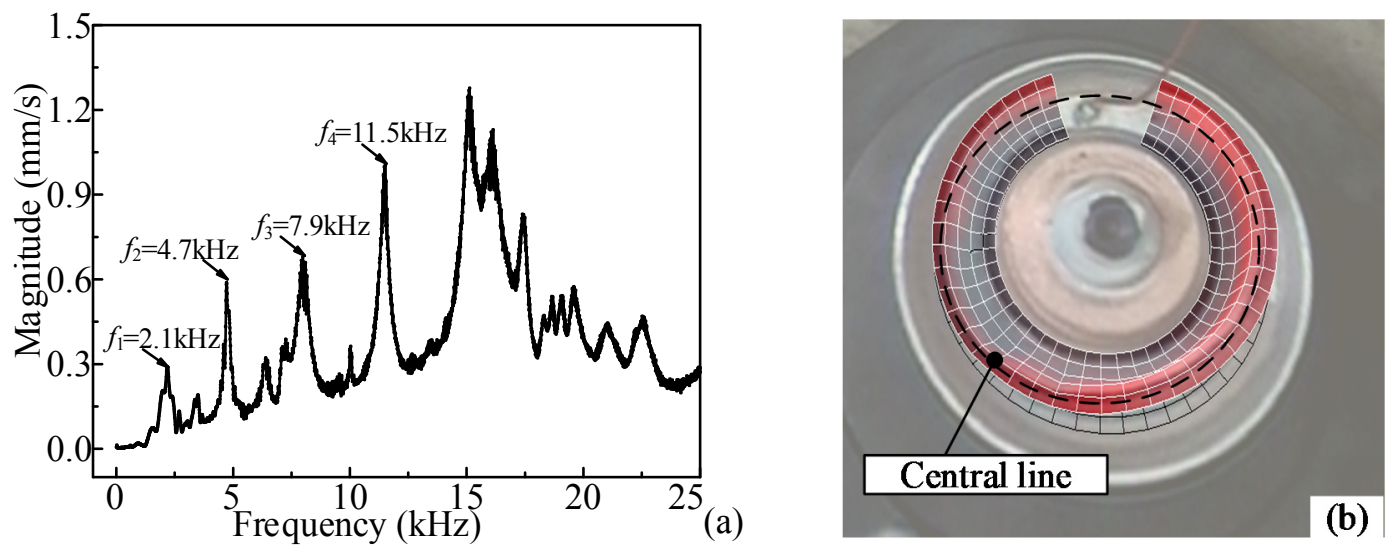

Figure 15. The vibration measurement results of vibrator: (a) the vibration velocity response spectrum; (b) the first order vibration shape.

Comparing the test results of the first four natural frequencies with the simulations, as shown in Table 3, we can see that the relative errors were in an acceptable range. Furthermore, the test result of the first-order vibration shape was very close to the simulation, when comparing Figure 15b with Figure 4a. It proved the correctness and feasibility of the numerical simulation methods, and that the methods can be used in future research to predict the working frequency of the micro-jet in advance. 
Table 3. Comparison of experimental and simulation results.

\begin{tabular}{ccccc}
\hline Resonance Frequency & $f_{1}$ & $f_{2}$ & $f_{3}$ & $f_{4}$ \\
\hline Experimental results $(\mathrm{kHz})$ & 2.1 & 4.7 & 7.9 & 11.5 \\
Simulation results $(\mathrm{kHz})$ & 1.83 & 4.32 & 7.01 & 9.49 \\
Relative error $(\%)$ & 12.86 & 8.09 & 11.27 & 17.48 \\
\hline
\end{tabular}

\subsection{Experiment Study of the Injection Performance}

The experimental platform for the study of injection performance mainly consisted of a waveform generator (RIGOL DG1022), a power amplifier and a digital storage oscilloscope (Tektronix TDS2012C), as shown in Figure 16.

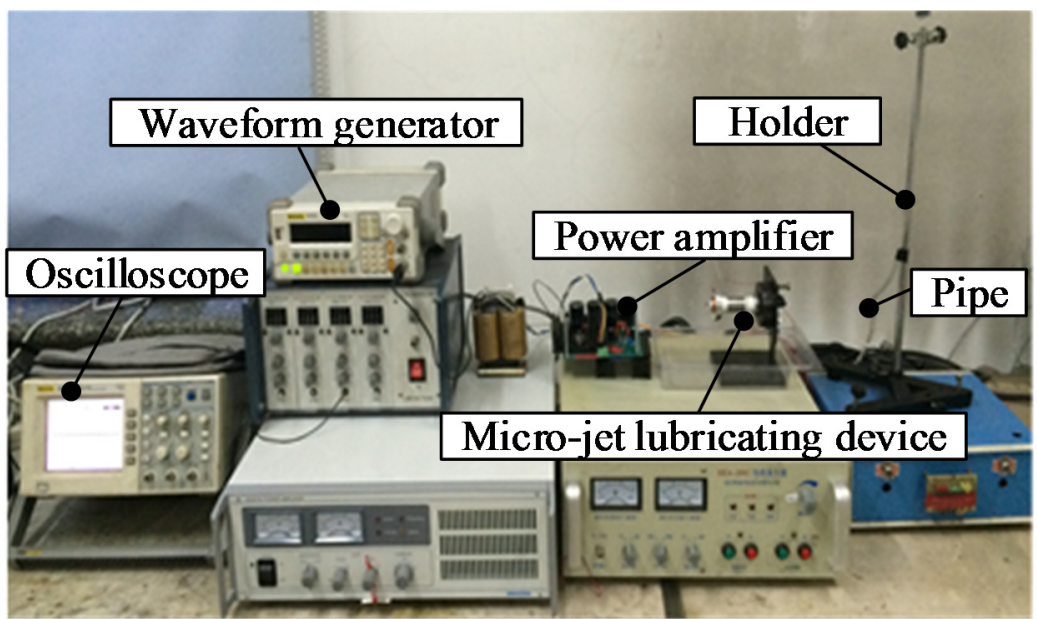

Figure 16. The experimental platform for the study of injection performance.

In practical application, what we are mainly focused on is the actual injection state of the micro-jet and the volume of the lubricating oil droplet under single-pulse voltage. We found that the smaller the volume of the droplet is, the longer the effective working time of the lubricating system will be under the condition of a limited lubricating oil supply. Therefore, the injection states and the volume of single droplets of the lubricating system under different pulse voltage excitations were analyzed in the experiments.

The lubricating oil was stored in the pipe, which, unlike the oil storage cavity, and the volume of a single droplet, was calculated with Equation (6), where $V$ is the volume of the single droplet, $D$ is the diameter of the pipe, $\Delta h_{0}$ is the drop height of the oil surface in the pipe, $f$ is the frequency of the pulse voltages, and $t$ is the time interval.

$$
V=\frac{\pi D^{2} \Delta h_{0}}{4 f t} \times 10^{-3}
$$

\subsubsection{The Influence of the Amplitude}

Setting the working frequency at $2.1 \mathrm{kHz}$, which was obtained in a previous test, the experimental injection states of the micro-jet under pulse voltages with different amplitude were obtained, as shown in Figure 17, when the duty ratio was set to $50 \%$. 

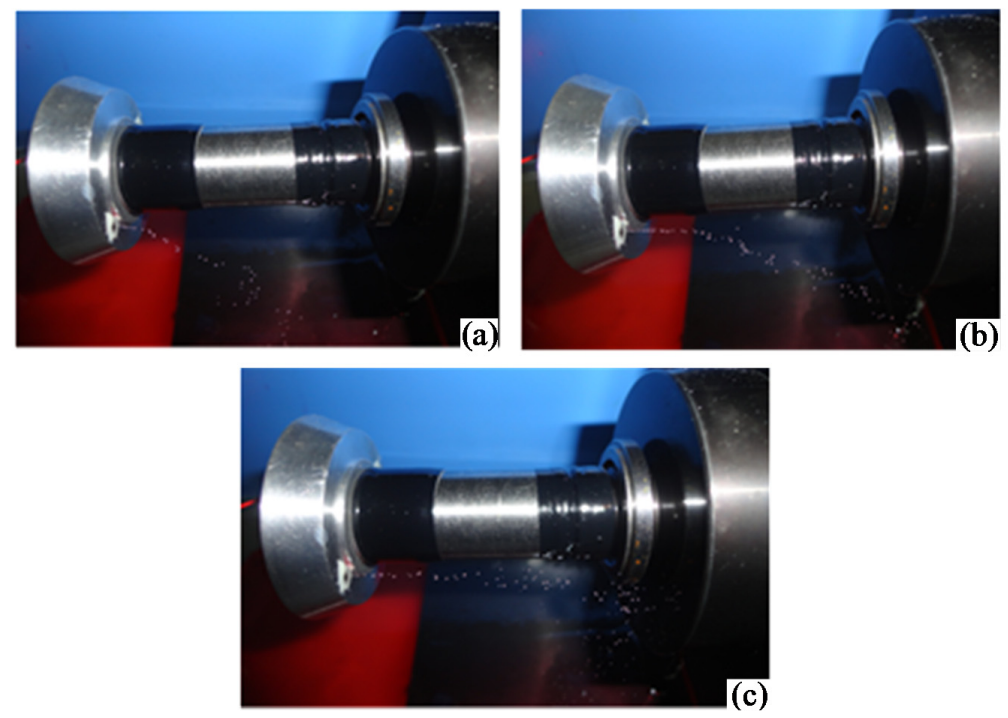

Figure 17. The injection states of the micro-jet under pulse voltages with different excitations: (a) the amplitude is $60 \mathrm{~V}$; (b) the amplitude is $80 \mathrm{~V}$; (c) the amplitude is $100 \mathrm{~V}$.

As we can see in Figure 17, the lateral injection distance and the level of the dispersion of the droplets all increased gradually along with the rising amplitudes of the pulse voltages, which was in agreement with the simulation results. The reason is that the acoustic pressure at the nozzle part increases when the amplitude rises, which results in the increase in the kinetic energy in the droplets. Furthermore, due to the interaction of the air and droplets, the greater the speed of the droplets is, the more intense the dispersion will be.

The relationship curve of the single droplet's volume and the amplitude of pulse voltages is shown in Figure 18. It appears that the volume increased slowly before $80 \mathrm{~V}$ and then changed quickly, which means that the influences of the amplitude were enhanced after $80 \mathrm{~V}$. The minimum volume of the droplets was $3.53 \times 10^{-6} \mathrm{~mL}$ when the amplitude of the pulse voltages was set to $60 \mathrm{~V}$.

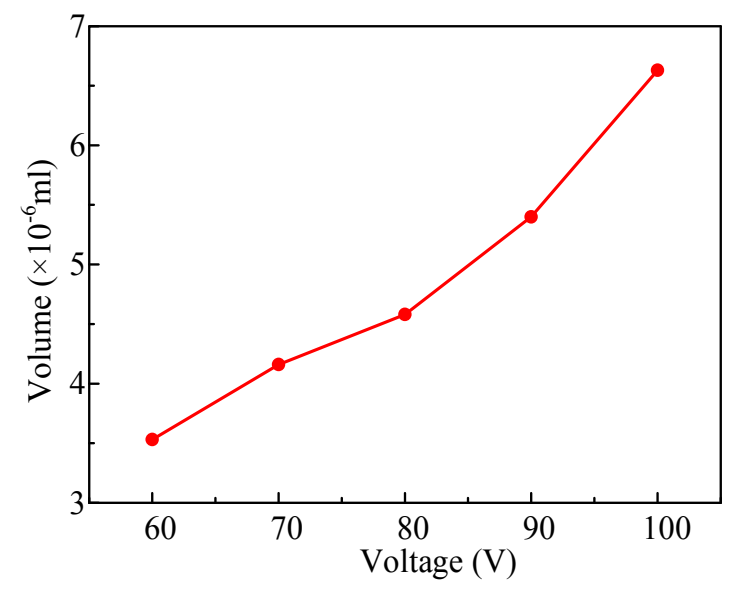

Figure 18. The relationship curve of droplet's volume and amplitude.

We concluded that, in order to increase the effective distance and the uniformity of lubrication, the amplitude of the pulse voltages should be increased. However, in order to increase the effective working time of the lubricating system under the condition of a limited lubricating oil supply, the amplitude of the pulse voltages should be decreased. Therefore, the appropriate amplitude should be chosen according to application situations and requirements. 


\subsubsection{The Influence of the Duty Ratio}

When the working frequency and amplitude were set to $2.1 \mathrm{kHz}$ and $100 \mathrm{~V}$ respectively, the experimental injection states of the micro-jet under pulse voltages with different duty ratios were obtained, as shown in Figure 19. With the rising duty ratio of the pulse voltages, the lateral injection distance and the level of the dispersion of the droplets all increased gradually, which corresponded to the results of the simulation. In addition, through these experiments, we found that the micro-jet cannot work properly when the duty ratio of the pulse voltages is less than $20 \%$ or more than $70 \%$. The main reason is that the mechanical responses of the vibrator requires a certain time; if a duty ratio of the pulse voltages is too high or too low, the vibrator will be unable to deform or return to its natural state.

Figure 20 shows the relationship between a single droplet's volume and the duty ratio of pulse voltages. As we can see, the volume increases slowly with the increase of the duty ratio, but the change gradually slows down. The minimum volume of the droplets is $5.14 \times 10^{-6} \mathrm{~mL}$ when the duty ratio of the pulse voltages is set to $30 \%$.

Therefore, for better uniformity of lubrication and longer effective distance, the higher duty ratio should be selected, but the duty ratio should be limited to less than $70 \%$. Furthermore, in order to increase the effective working time of the lubricating system, the lower duty ratio should be selected, but the duty ratio should exceed $20 \%$. Additionally, the appropriate amplitude should be chosen according to application situations and requirements.
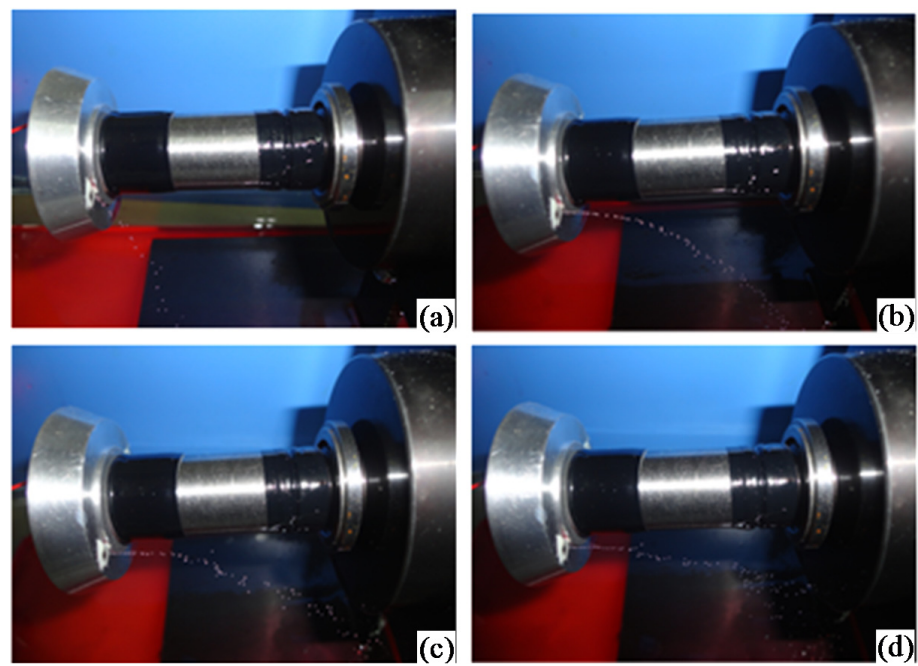

Figure 19. The injection states of the micro-jet under pulse voltages with different duty ratios: (a) the duty ratio is $30 \%$; (b) the duty ratio is $40 \%$; (c) the duty ratio is $50 \%$; (d) the duty ratio is $60 \%$.

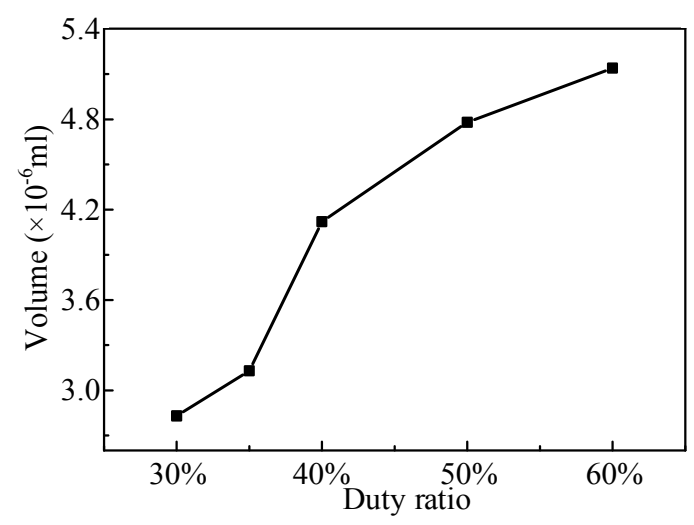

Figure 20. The relationship between single droplet's volume and the duty ratio. 


\section{Conclusions}

A novel piezoelectric micro-jet was designed and embedded in the bearing system to supply the DOD lubrication. The mathematic model of velocity-inlet boundary conditions was deduced and applied to the injection performance simulations to analyze the deformations and motion states of the droplets, which could not be measured and observed through experiments. The molding time, velocity, molding length, and dispersion condition of the droplets under different pulse voltages were analyzed, and, to obtain better performance of the micro-jet, the appropriate excitation parameters were given in accordance with the simulation results.

The actual working frequency and injection states of the micro-jet under different excitations were obtained by the experiments and the volumes of the droplets were calculated. The experiments proved that the piezoelectric micro-jet lubricating system can work effectively and that the methods of adjusting the injection performance to meet different requirements are given according to the analysis of the experimental results.

The validity of the mathematic model of velocity-inlet boundary conditions was confirmed through the comparison between simulations and experiments; thus, the injection performance of the micro-jet can be predicted by the injection simulation method described in this paper. In conclusion, this paper provides an effective solution to the problem of bearing lubrication.

Based on this, we will carry out complete multiple physical field coupling simulations and experimental validations of the coupled model in future studies so that the injection performance of the micro-jet for lubrication can be further analyzed.

Acknowledgments: This project was supported by the National Natural Science Foundation of China (51075082, 51375107).

Author Contributions: All authors conceived and designed the experiments and analyzed the data; all authors contributed to the writing of the paper.

Conflicts of Interest: The authors declare no conflict of interest.

\section{References and Notes}

1. Heinzl, J.; Hertz, C.H. Ink-jet printing. In Advances in Electronics and Electron Physics; Peter, W.H., Ed.; Academic Press: Cambridge, MA, USA, 1985; Volume 65, pp. 91-171.

2. He, M.W.; Sun, L.L.; Hu, K.Y.; Zhu, Y.L.; Chen, H.N. Analysis of dod inkjet printhead performance for printable electronics fabrication using dynamic lumped element modeling and swarm intelligence based optimal prediction. J. Cent. South Univ. 2015, 22, 3925-3934. [CrossRef]

3. Stumpf, F.; Schoendube, J.; Gross, A.; Rath, C.; Niekrawietz, S.; Koltay, R.; Roth, G. Single-cell pcr of genomic DNA enabled by automated single-cell printing for cell isolation. Biosens. Bioelectron. 2015, 69, 301-306. [CrossRef] [PubMed]

4. Narayan, R.; Stafslien, S.; VanderWal, L.; Boehm, R.; Petrochenko, P.; Nasir, A. Use of piezoelectric inkjet printing to apply antifungal agents to transdermal drug delivery devices. J. Am. Acad. Dermatol. 2015, 72, AB139.

5. Uddin, M.J.; Scoutaris, N.; Klepetsanis, P.; Chowdhry, B.; Prausnitz, M.R.; Douroumis, D. Inkjet printing of transdermal microneedles for the delivery of anticancer agents. Int. J. Pharm. 2015, 494, 593-602. [CrossRef] [PubMed]

6. Boehm, R.D.; Daniels, J.; Stafslien, S.; Nasir, A.; Lefebvre, J.; Narayan, R.J. Polyglycolic acid microneedles modified with inkjet-deposited antifungal coatings. Biointerphases 2015, 10. [CrossRef] [PubMed]

7. Mazzalai, A.; Balma, D.; Chidambaram, N.; Jin, L.; Muralt, P.; IEEE. Simultaneous piezoelectric and ferroelectric characterization of thin films for mems actuators. In Proceedings of the 2013 IEEE International Symposium on the Applications of Ferroelectric and Workshop on the Piezoresponse Force Microscopy (Isaf/Pfm), Prague, Czech Republic, 21-25 July 2013; pp. 363-365.

8. Senousy, M.S.; Li, F.X.; Mumford, D.; Gadala, M.; Rajapakse, R. Thermo-electro-mechanical performance of piezoelectric stack actuators for fuel injector applications. J. Intell. Mater. Syst. Struct. 2009, 20, 387-399. [CrossRef] 
9. Glynne-Jones, P.; Coletti, M.; White, N.M.; Gabriel, S.B.; Bramanti, C. A feasibility study on using inkjet technology, micropumps, and mems as fuel injectors for bipropellant rocket engines. Acta Astronaut. 2010, 67, 194-203. [CrossRef]

10. Voevodin, A.A.; O’Neill, J.P.; Zabinski, J.S. Nanocomposite tribological coatings for aerospace applications. Surf. Coat. Technol. 1999, 116-119, 36-45. [CrossRef]

11. Vattikuti, S.V.P.; Byon, C.; Reddy, C.V.; Venkatesh, B.; Shim, J. Synthesis and structural characterization of mos2 nanospheres and nanosheets using solvothermal method. J. Mater. Sci. 2015, 50, 5024-5038. [CrossRef]

12. Fan, X.Q.; Wang, L.P.; Li, W.; Wan, S.H. Improving tribological properties of multialkylated cyclopentanes under simulated space environment: Two feasible approaches. ACS Appl. Mater. Interfaces 2015, 7, 14359-14368. [CrossRef] [PubMed]

13. Zhang, S.W.; Hu, L.T.; Qiao, D.; Feng, D.P.; Wang, H.Z. Vacuum tribological performance of phosphonium-based ionic liquids as lubricants and lubricant additives of multialkylated cyclopentanes. Tribol. Int. 2013, 66, 289-295. [CrossRef]

14. Sathyan, K.; Hsu, H.Y.; Lee, S.H.; Gopinath, K. Development of a centrifugal oil lubricator for long-term lubrication of spacecraft attitude control systems-experimental evaluation. Tribol. Trans. 2011, 54, 832-839. [CrossRef]

15. Mohammadpour, M.; Johns-Rahnejat, P.M.; Rahnejat, H. Roller bearing dynamics under transient thermal-mixed non-newtonian elastohydrodynamic regime of lubrication. Proc. Inst. Mech. Eng. KJ. Multi-body Dyn.. 2015, 229, 407-423. [CrossRef]

(C) 2016 by the authors; licensee MDPI, Basel, Switzerland. This article is an open access article distributed under the terms and conditions of the Creative Commons by Attribution (CC-BY) license (http:/ / creativecommons.org/licenses/by/4.0/). 